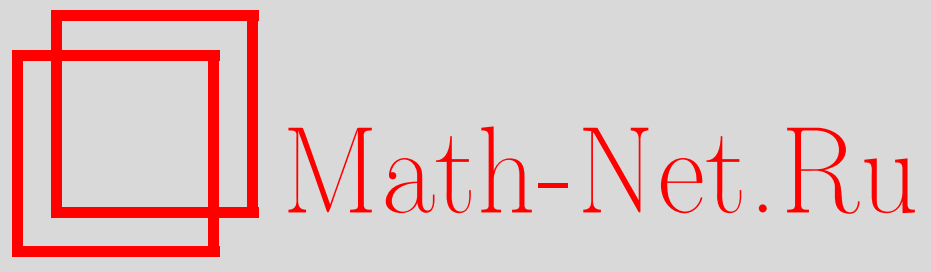

Д. Б. Питтс, У. К. Шив, Массивная гравитация с универсальным взаимодействием, ТМФ, 2007, том 151, номер 2, 311-336

DOI: https://doi.org/10.4213/tmf6047

Использование Общероссийского математического портала Math-Net.Ru подразумевает, что вы прочитали и согласны с пользовательским соглашением http://www . mathnet.ru/rus/agreement

Параметры загрузки:

IP : 52.87 .193 .239

26 апреля 2023 г., 12:41:37

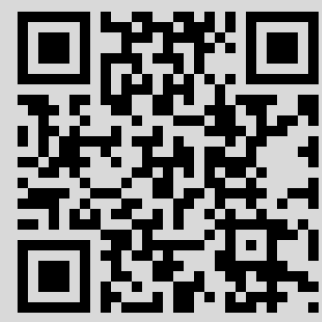




\section{МАССИВНАЯ ГРАВИТАЦИЯ С УНИВЕРСАЛЬНЫМ ВЗАИМОДЕЙСТВИЕМ}

Из линейной теории в плоском пространстве-времени с использованием калибровочной инвариантности свободных полей и универсального взаимодействия выводятся уравнения Эйнштейна. Гравитационный потенциал может быть как ковариантым, так и контравариантным и являться весом почти любой плотности. Эти результаты применяются для получения массивных вариантов уравнений Эйнштейна с универсальным взаимодействием, приводящих к двум однопараметрическим семействам различных теорий со спином 2 и спином 0. Таким образом, теория Френда-Махешвари-Шонберга, несмотря на ее выделенность в некоторых отношениях, не является единственным массивным обобщением теории Эйнштейна с универсальным взаимодействием. Полученные теории составляют подмножество теорий, найденных Огиевецким и Полубариновым в рамках другого подхода. Вопрос о положительности энергии, который продолжает обсуждаться, в случае сферической симметрии можно рассмотреть численно. Сделано несколько замечаний, касающихся причинности в случае двух наблюдаемых метрик и необходимости калибровочного произвола. Рассмотрены некоторые критические замечания, высказанные Падманабаном по поводу полевого вывода уравнений, подобных уравнениям Эйнштейна.

Ключевые слова: массивная гравитация, биметрика, духи, положительная масса, причинность.

\section{1. ВВЕДЕНИЕ}

Построение релятивистской теории гравитации на основе таких принципов, как аналогия с теорией электромагнетизма Максвелла, универсальное взаимодействие гравитационного поля с объединенным комплексом гравитации-материи и энергииимпульса, а также требование, чтобы только одни уравнения гравитационного поля (без уравнений материи, по аналогии с сохранением электромагнитного заряда) обусловливали сохранение энергии-импульса, составляло основное содержание работы

${ }^{*}$ Department of Physics, The University of Texas at Austin, USA

${ }^{\dagger}$ Department of Mathematics, St. Edward's University, Austin, Texas; History and Philosophy of Science Graduate Program, University of Notre Dame, Notre Dame, Indiana, USA. E-mail: jpitts@nd.edu 
Эйнштейна по поиску адекватной теории гравитации в 1913-1915 гг. (см. [1], [2]). Впоследствии Эйнштейн снизил значение этих исследований [2], и указанные идеи позднее стали ассоциироваться с неэйнштейновским теоретико-полевым подходом к гравитации. При таком выводе уравнений Эйнштейна или подобных им уравнений гравитационного поля можно использовать априори предпочтительные координаты и канонический тензор энергии-импульса или плоскую фоновую метрику и вариационный метрический тензор энергии-импульса, при этом различия между последними в основном формальные.

Ряд авторов [3]-[14] обсуждали полезность применимости фоновой метрики $\eta_{\mu \nu}$ в общей теории относительности или возможность вывода этой теории, приближенно или точно, из теории в плоском пространстве-времени ${ }^{1)}$. Фоновая метрика позволяет ввести гравитационный тензор энергии-импульса [17], а не просто псевдотензор. В результате гравитационные энергия и импульс координатно независимы, но зависят от калибровки [18]. Если мы хотим всерьез рассматривать фоновую метрику как свойство пространства-времени, а не относиться к ней, как к полезной выдумке, то следует рассмотреть соотношение между нулевыми конусами эффективной искривленной метрики и плоской фоновой метрики. Этот вопрос не без успеха изучался для случая безмассовых уравнений Эйнштейна [13].

В качестве подготовки к рассмотрению массивных теорий гравитации мы обобщим наш вывод уравнений Эйнштейна с использованием калибровочной инвариантности для свободного поля и универсального взаимодействия [12] таким образом, чтобы сделать его пригодным в случае плотности практически любого веса как для ковариантной, так и для контравариантной валентности симметричного гравитационного потенциала ранга 2. Эта общность до некоторой степени параллельна общности классических работ Крейчнана [4], но наш вывод содержит несколько усовершенствований, и его легко приспособить к массивным теориям. Разный выбор расположения индексов и веса плотности не приводит ни к каким различиям в безмассовых теориях (после переопределения полей), однако в настоящей работе разные способы такого выбора дают различные массивные теории. Попутно мы рассмотрим несколько возражений со стороны Падманабана, высказанных им недавно по поводу полевых выводов уравнений Эйнштейна [19].

В последнее время вопрос о массивной гравитации с компонентами спина 2 и спина 0 обсуждался несколькими авторами [20], [21]. Хотя и допустимо просто постулировать нелинейные свойства (если таковые имеются) массового члена, предпочтительно найти хорошо мотивированные теоретические принципы, ограничивающие

1) Заметим, что известен также вывод общей теории относительности из самодействия на искривленном фоне [10], [15]. Исследовалась и возможность (или ее отсутствие) безмассовых мультигравитонных теорий, подобных теориям Янга-Миллса [16]. Кроме того, интересно рассмотреть теории на фоне пространств (A)dS, теории, нарушающие лоренц-инвариантность, и теории ЧернаСаймонса с топологическим массивным членом. Однако мы рассмотрим только наиболее традиционную задачу о массивной гравитации. Мы изучаем четырехмерное пространство-время, но выводы должны непосредственно обобщаться на любую (целую) размерность не ниже трех. 
такой выбор. Некоторое время назад Огиевецкий и Полубаринов [7] вывели двухпараметрическое семейство массивных вариантов уравнений Эйнштейна, содержащее оба наши однопараметрические семейства (квантование кратко рассматривалось в работе [22]). Их вывод был основан на калибровочной инвариантности (по крайней мере, для безмассовой части плотности лагранжиана), а не на универсальном взаимодействии. Вместо этого они использовали принцип ограничения по спину, чтобы исключить из полной нелинейной теории с взаимодействием некоторые степени свободы, многие из которых имели неправильный знак и, следовательно, отрицательную энергию. Массивная теория Фройнда-Махешвари-Шонберга (ФМШ) была первоначально выведена с использованием универсального взаимодействия с каноническим, а не с метрическим тензором энергии-импульса [23]. Хотя теория ФМШ воспроизводится исходя из наших результатов, она, несмотря на имеющиеся утверждения [23], [24], не является единственным массивным вариантом уравнений Эйнштейна с универсальным взаимодействием. Наш вывод с использованием метрического тензора энергии-импульса выглядит более коротким и более ясным, чем вывод теории ФМШ с использованием канонического тензора энергии-импульса. В следующих работах мы рассмотрим универсальное взаимодействие, используя тетрадный формализм вместо метрического, что даст дополнительные теории с универсальным взаимодействием, и с помощью метрического формализма покажем, что все теории Огиевецкого-Полубаринова являются теориями с универсальным взаимодействием. Все известные теории с универсальным взаимодействием соответствуют теориям из семейства Огиевецкого-Полубаринова, так что их вывод и вывод универсального метрического взаимодействия в настоящее время дают совпадающие результаты. Теория ФМШ, впоследствии принятая Логуновым с соавторами [11], сталкивается также с вопросами о положительности энергии и причинности, по поводу которых ниже будет сделано несколько замечаний.

\section{2. ЭФФЕКТИВНО ГЕОМЕТРИЧЕСКИЕ ТЕОРИИ С УНИВЕРСАЛЬНЫМ ВЗАИМОДЕЙСТВИЕМ И КАЛИБРОВОЧНОЙ ИНВАРИАНТНОСТЬЮ}

Ранее мы выводили теорию Эйнштейна и другие эффективно геометрические теории, используя калибровочно-инвариантные свободно-полевые теории и универсальное взаимодействие [12], в значительной мере на основании работ Крейчнана [4] и Дезера [9]. Если ранее гравитационный потенциал выбирался в виде симметричного ковариантного тензорного поля ранга 2, то теперь мы обобщим этот вывод для случая плотности почти любого веса, ковариантной или контравариантной валентности. С учетом общности вывода Крейчнана неудивительно, что эти обобщения снова приводят лишь к теории Эйнштейна и другим эффективно геометрическим теориям. Для безмассовых теорий это не дает ничего существенно нового, но ниже эти выводы будут впервые применены к массивной гравитации. 


\section{1. Действие свободного поля для потенциала ковариантной тензор-} ной плотности. Для безмассовых теорий предполагается исходная инфинитезимальная инвариантность (с точностью до граничных членов) свободного гравитационного действия. Для последующего вывода массивных теорий калибровочная свобода будет нарушена естественным массовым членом, алгебраически зависящим от полей, но члены с производными сохранят калибровочную инвариантность.

Пусть $S_{\mathrm{f}}$ - действие свободной симметричной тензорной плотности $\tilde{\gamma}_{\mu \nu}$ (с весом плотности $-l$, где $l \neq 1 / 2)$ в пространстве-времени с плоским метрическим тензором $\eta_{\mu \nu}$ в произвольных координатах. Согласованная с метрикой ковариантная производная без кручения обозначается как $\partial_{\mu}$, так что $\partial_{\alpha} \eta_{\mu \nu}=0$. Удобно использовать не саму плоскую метрику, а связанную с ней невырождаемую метрику $\tilde{\eta}_{\mu \nu}=\eta_{\mu \nu}(\sqrt{-\eta})^{-l}$ с весом $-l$. Заметим, что в запрещенном случае $l=1 / 2$ метрика $\tilde{\eta}_{\mu \nu}$ теряет обратимость: $\eta_{\mu \nu}(\sqrt{-\eta})^{-1 / 2}$ определяет только нулевой конус, а не весь метрический тензор ${ }^{2)}$. Оказывается, что поле $\tilde{\gamma}_{\mu \nu}$ становится гравитационным потенциалом. В работах по петлевой квантовой гравитации стало привычным обозначать вес плотности для поля соответствующим числом тильд над или под символом (для выражения положительности или отрицательности веса плотности), но в данной работе это невозможно. Вес плотности $l(l \neq 1 / 2)$ может быть большим, нецелым или даже иррациональным, так что мы просто будем отмечать сверху тильдой большинство плотностей. Все индексы поднимаются и опускаются с помощью соответственно тензоров $\eta^{\mu \nu}$ и $\eta_{\mu \nu}$, за двумя исключениями. Для превращенной в плотность плоской метрики $\tilde{\eta}_{\mu \nu}$ противоположным образом превращенная в плотность обратная плоская метрика есть $\tilde{\eta}^{\mu \nu}$. Аналогично метрика, обратная к превращенной в плотность искривленной метрике $\tilde{g}_{\mu \nu}$, которая определяется ниже, есть $\tilde{g}^{\mu \nu}$. Поэтому мы будем интенсивно использовать тензорные плотности, нелишне напомнить вид их ковариантных производных и производных Ли. $(1,1)$-плотность $\tilde{\phi}_{\beta}^{\alpha}$ веса $w$ дает представительный пример. Производная Ли и $\eta$-ковариантная производная имеют вид соответственно [25]

$$
\begin{aligned}
& £_{\xi} \tilde{\phi}_{\beta}^{\alpha}=\xi^{\mu} \tilde{\phi}_{\beta}^{\alpha, \mu}-\tilde{\phi}_{\beta}^{\mu} \xi^{\alpha},{ }_{\mu}+\tilde{\phi}_{\mu}^{\alpha} \xi^{\mu}{ }_{, \beta}+w \tilde{\phi}_{\beta}^{\alpha} \xi^{\mu},{ }_{\mu}, \\
& \partial_{\mu} \tilde{\phi}_{\beta}^{\alpha}=\tilde{\phi}_{\beta}^{\alpha}, \mu+\tilde{\phi}_{\beta}^{\sigma} \Gamma_{\sigma \mu}^{\alpha}-\tilde{\phi}_{\sigma}^{\alpha} \Gamma_{\beta \mu}^{\sigma}-w \tilde{\phi}_{\beta}^{\alpha} \Gamma_{\sigma \mu}^{\sigma} \text {, }
\end{aligned}
$$

где $\Gamma_{\beta \mu}^{\sigma}$ - символы Кристоффеля для $\eta_{\mu \nu}$. Как только мы определим искривленную метрику $g_{\mu \nu}$, мы получим аналогичную $g$-ковариантную производную $\nabla$ с символами Кристоффеля $\left\{\begin{array}{l}\alpha \\ \sigma \mu\end{array}\right\}$.

Желание избежать духов мотивирует калибровочную инвариантность в линейных теориях [26]. Мы требуем, чтобы свободно-полевое действие $S_{\mathrm{f}}$ при инфинитезимальном калибровочном преобразовании

$$
\tilde{\gamma}_{\mu \nu} \rightarrow \tilde{\gamma}_{\mu \nu}+\delta \tilde{\gamma}_{\mu \nu}, \quad \delta \tilde{\gamma}_{\mu \nu}=\partial_{\mu} \tilde{\xi}_{\nu}+\partial_{\nu} \tilde{\xi}_{\mu}+c \eta_{\mu \nu} \partial^{\alpha} \tilde{\xi}_{\alpha}
$$

2)Этот исключительный случай представлял интерес при выводе слабо биметрических теорий [12], в которых величина $\sqrt{-\eta}$ может появляться в полевых уравнениях теорий с взаимодействием. 
изменялось только на граничные члены, причем $c \neq-1 / 2^{3)}$, а $\tilde{\xi}_{\nu}$ - поле произвольной ковекторной плотности веса $-l$. Можно предвидеть появление связи между $l$ и $c$.

Для всякого действия $S_{\mathrm{f}}$, инвариантного в этом смысле относительно $(3)$, некоторая линейная комбинация свободно-полевых уравнений оказывается, как мы сейчас покажем, тождественно бездивергентной. Действие изменяется на

$$
\delta S_{\mathrm{f}}=\int d^{4} x\left[\frac{\delta S_{\mathrm{f}}}{\delta \tilde{\gamma}_{\mu \nu}}\left(\partial_{\nu} \tilde{\xi}_{\mu}+\partial_{\mu} \tilde{\xi}_{\nu}+c \eta_{\mu \nu} \partial^{\alpha} \tilde{\xi}_{\alpha}\right)+e^{\mu}, \mu\right]=\int d^{4} x f^{\mu},_{\mu} .
$$

Явный вид граничных членов, а именно $e^{\mu},{ }_{\mu}$ и $f^{\mu},{ }_{\mu}$, нам не нужен. Интегрируя по частям, выбирая для $\tilde{\xi}_{\mu}$ компактный носитель с целью уничтожения граничных членов и используя произвол в $\tilde{\xi}_{\mu}$, получаем тождество

$$
\partial_{\mu}\left(\frac{\delta S_{\mathrm{f}}}{\delta \tilde{\gamma}_{\mu \nu}}+\frac{c}{2} \eta^{\mu \nu} \eta_{\sigma \alpha} \frac{\delta S_{\mathrm{f}}}{\delta \tilde{\gamma}_{\sigma \alpha}}\right)=0 .
$$

Это обобщенное тождество Бьянки для свободной теории, которое в наиболее распространенном случае является линеаризованным вариантом исходного геометрического тождества Бьянки. Естественный выбор для $S_{\mathrm{f}}$ состоит в линеаризованной плотности лагранжиана ОТО, но столь подробных допущений о виде $S_{\mathrm{f}}$ не использовалось. Поскольку невзаимодействующие поля без источников ненаблюдаемы [27], интересную теорию можно получить только после введения взаимодействия.

Как в ответ Эйнштейну давно уже указывал Кречман [28]-[31], любой теории можно дать общековариантную формулировку в том смысле, что уравнения будут выполняться в любой системе координат, декартовой или нет. Получающуюся таким образом формулировку можно назвать слабо или тривиально общековариантной. Достижение слабой общей ковариантности часто связано с использованием абсолютных объектов [29], таких как плоский метрический тензор, как это сделано в данной работе. Существенно новый аспект теории гравитации Эйнштейна состоит, как считается, в отсутствии абсолютных объектов или "предгеометрии" [29], [32] $]^{4)}$. Это свойство можно назвать сильной или нетривиальной общей ковариантностью. Указанное различие между двумя пониманиями общей ковариантности обсуждалось ранее в работах [35]. Вывод, представленный здесь и в некоторых других работах, следует из слабой общековариантной теории и приводит к сильной общековариантной теории, без абсолютных объектов ${ }^{5)}$. Таким образом, не следует принимать вводящих в заблуждение утверждений [19] о том, что вывод уравнений

\footnotetext{
3) Случай $c=-1 / 2$ дает просто скалярную теорию в работе Огиевецкого и Полубаринова [7].

4) В действительности дело обстоит более сложным образом: Герох и Джулини недавно отметили тот факт, что определитель метрического тензора $g$ является абсолютным объектом, поскольку всякая точка имеет такую окрестность и систему координат к ней, что компонента $g$ имеет значение -1 [33], [34].

5) То обстоятельство, что $g$ считается абсолютным в общей теории относительности в рамках программы абсолютных объектов Андерсона-Фридмана, указывает, что на самом деле общая теория относительности не является сильно общековариантной. Но остается в силе, что здесь присутствуют два весьма разных понятия общей ковариантности. Кроме полевых уравнений, при
} 
Эйнштейна на основе плоской модели пространства-времени приводит к общей ковариантности только потому, что она заложена с самого начала. Эти два понимания общей ковариантности весьма различны, так что критические замечания Падманабана оказываются ошибочными, поскольку приводят к двусмысленности.

2.2. Метрическая тензорная плотность энергии-импульса. Если тензору энергии-импульса надлежит быть источником гравитационного потенциала $\tilde{\gamma}_{\mu \nu}$, то внутренняя согласованность требует, чтобы использовался полный тензор энергииимпульса, включающий гравитационные энергию и импульс, а не только негравитационные ("материальные"), поскольку только полный тензор энергии-импульса является бездивергентным в смысле $\partial_{\nu}[9]$, или, что эквивалентно, в смысле декартовой координатной дивергенции. Для получения глобального закона сохранения требуется обращение в нуль координатной расходимости 4-тока.

Используя метрическое предписание [4], [17], [29], [36], можно вывести из действия $S$ выражение для полной тензорной плотности энергии-импульса следующим образом. Действие зависит от плоской метрической плотности $\tilde{\eta}_{\mu \nu}$, гравитационного потенциала $\tilde{\gamma}_{\mu \nu}$ и полей материи $u$. При этом $u$ представляет собой произвольный набор динамических тензорных полей произвольного ранга, произвольного расположения индексов и произвольного веса плотности. Если использовать спинорный формализм Огиевецкого и Полубаринова, в который входит квадратный корень из метрики, а не тетрада или другая дополнительная структура [37], то, по-видимому, окажется возможным включить и спинорные поля.

При произвольном инфинитезимальном преобразовании координат, описываемом векторным полем $\xi^{\mu}$, действие изменяется на величину

$$
\delta S=\int d^{4} x\left(\frac{\delta S}{\delta \tilde{\gamma}_{\mu \nu}} £_{\xi} \tilde{\gamma}_{\mu \nu}+\frac{\delta S}{\delta u} £_{\xi} u+\frac{\delta S}{\delta \tilde{\eta}_{\mu \nu}} £_{\xi} \tilde{\eta}_{\mu \nu}+g^{\mu}, \mu\right),
$$

причем граничные члены из $g^{\mu}, \mu$ обращаются в нуль из-за компактности носителя $\xi^{\mu}$. Однако $S$ - скаляр, поэтому $\delta S=0$. Полагая, что уравнения для материи и гравитационного поля выполняются, интегрируя по частям, отбрасывая обращающиеся в нуль граничные члены и используя произвол в определении векторного поля $\xi^{\mu}$, получаем

$$
\partial_{\nu}\left(\frac{\delta S}{\delta \tilde{\eta}_{\mu \nu}}-\frac{l}{2} \tilde{\eta}_{\alpha \beta} \tilde{\eta}^{\mu \nu} \frac{\delta S}{\delta \tilde{\eta}_{\alpha \beta}}\right)=0
$$

Эта величина является плотностью тензора энергии-импульса для полей материи и гравитации. При взятии функциональной производной $\delta S / \delta \tilde{\eta}_{\mu \nu}$ условие того, что метрика $\eta_{\mu \nu}$ является плоской, ослабляется, а затем восстанавливается. Иногда на этот факт указывают, критикуя теорию [19]. Однако подобную процедуру нельзя

поиске абсолютных объектов следует также расмотреть топологию, граничные условия и причинность [13]. Подчеркнем, что мы рассматриваем безмассовые случаи; очевидно, что массивные теории содержат плоскую метрику и потому не являются сильно общековариантными. 
отвергать даже в случае плоского пространства-времени, поскольку они представляют собой просто формальный прием, полезный при определении тензора энергииимпульса, а не неправомерное использование искривленного пространства-времени. Используя связь между тензором энергии-импульса Розенфельда и симметризованным каноническим тензором энергии-импульса Бельинфанте [36], [38], можно рассматривать метрическое предписание как математически более короткий путь к концептуально безупречному, но математически неудобному тензору Бельинфанте, модифицированному членами, пропорциональными уравнениям движения. На этом этапе метрический тензор энергии-импульса не является единственным, поскольку можно добавить члены, пропорциональные уравнениям движения и их производным, а также суперпотенциалам. Другую возможность могли бы дать использование лагранжевых множителей и допущение для фоновой метрики быть плоской только на массовой оболочке [39]. Как будет показано ниже, разумное использование произвола в выборе суперпотенциала играет важную роль в выводе полевых уравнений.

2.3. Полное действие с универсальным взаимодействием. Найдем действие $S$, подчиняющееся правдоподобному физическому постулату о том, что обратимая линейная комбинация уравнений Эйлера-Лагранжа должна быть просто обратимой линейной комбинацией свободных полевых уравнений для $S_{\mathrm{f}}$ с добавлением полного тензора энергии-импульса. Простой способ наложить это требование сводится к условию

$$
\frac{\delta S}{\delta \tilde{\gamma}_{\mu \nu}}=\frac{\delta S_{\mathrm{f}}}{\delta \tilde{\gamma}_{\mu \nu}}-\lambda \frac{\delta S}{\delta \tilde{\eta}_{\mu \nu}},
$$

где $\lambda=-\sqrt{32 \pi G}$. (Поскольку линейные комбинации свободных уравнений ЭйлераЛагранжа и полных уравнений Лагранжа с взаимодействием в данном случае обратимы, то эту линейную комбинацию можно применить и к тензору энергииимпульса.) Представляется разумным положить $c=-l$, для того чтобы обобщенное тождество Бьянки и тензорная плотность энергии-импульса приняли подобный вид.

Фундаментальными переменными в этом подходе являются гравитационный потенциал $\tilde{\gamma}_{\mu \nu}$ и плоская метрическая плотность $\tilde{\eta}_{\mu \nu}$. Однако в действии $S$ мы можем свободно переходить от переменных $\tilde{\gamma}_{\mu \nu}$ и $\tilde{\eta}_{\mu \nu}$ к биметрическим переменным $\tilde{g}_{\mu \nu}$ и $\tilde{\eta}_{\mu \nu}[4]$, где

$$
\tilde{g}_{\mu \nu}=\tilde{\eta}_{\mu \nu}-\lambda \tilde{\gamma}_{\mu \nu}
$$

(Тогда из $\tilde{g}_{\mu \nu}$ можно определить метрику $g_{\mu \nu}$ с помощью матричной алгебры и далее определить $g$-ковариантную производную $\nabla$ обычным образом. Нам, однако, не понадобится явно использовать $\nabla$.)

Приравнивая коэффициенты при вариациях, получаем уравнение

$$
\frac{\delta S}{\delta \tilde{\eta}_{\mu \nu}}\left|\tilde{\gamma}=\frac{\delta S}{\delta \tilde{\eta}_{\mu \nu}}\right| \tilde{g}+\frac{\delta S}{\delta \tilde{g}_{\mu \nu}}
$$


для $\delta \tilde{\eta}_{\mu \nu}$ и уравнение

$$
\frac{\delta S}{\delta \tilde{\gamma}_{\mu \nu}}=-\lambda \frac{\delta S}{\delta \tilde{g}_{\mu \nu}}
$$

для $\delta \tilde{\gamma}_{\mu \nu}$. Взятые вместе, эти результаты дают уравнение

$$
\lambda \frac{\delta S}{\delta \tilde{\eta}_{\mu \nu}}\left|\tilde{\gamma}=\lambda \frac{\delta S}{\delta \tilde{\eta}_{\mu \nu}}\right| \tilde{g}-\frac{\delta S}{\delta \tilde{\gamma}_{\mu \nu}} .
$$

Уравнение (12) разбивает тензор энергии-импульса на две части, одна из которых обращается в нуль, когда гравитация находится на массовой оболочке, а другая при этом в нуль не обращается. Использование этого результата в постулате универсального взаимодействия (8) дает

$$
\lambda \frac{\delta S}{\delta \tilde{\eta}_{\mu \nu}} \mid \tilde{g}=\frac{\delta S_{\mathrm{f}}}{\delta \tilde{\gamma}_{\mu \nu}} .
$$

Вычисляя дивергенцию, вспоминая тождество Бьянки (5) в свободной теории и используя равенство $c=-l$, находим

$$
\partial_{\mu}\left(\frac{\delta S}{\delta \tilde{\eta}_{\mu \nu}}\left|\tilde{g}-\frac{l}{2} \tilde{\eta}^{\mu \nu} \tilde{\eta}_{\alpha \beta} \frac{\delta S}{\delta \tilde{\eta}_{\alpha \beta}}\right| \tilde{g}\right)=0 .
$$

Величина в скобках есть точно $(\sqrt{-\eta})^{l} \delta S / \delta \eta_{\mu \nu} \mid \tilde{g}$. Таким образом, та часть тензора энергии-импульса, которая не пропорциональна уравнениям гравитационного поля, имеет тождественно нулевые дивергенции (по любому из индексов), т.е. является (симметричным) "ротором" [29]. Разбиение тензора энергии-импульса на части гарантирует, что в безмассовом случае уравнения гравитационного поля сами по себе, без какого бы то ни было независимого постулирования уравнений материи, приводят к сохранению энергии-импульса для получающихся в результате эффективно геометрических уравнений поля.

Поскольку величина $\delta S / \delta \eta_{\mu \nu} \mid \tilde{g}$ симметрична и имеет тождественно нулевую дивергенцию по любому индексу, она с необходимостью имеет вид [40]

$$
\frac{\delta S}{\delta \eta_{\mu \nu}} \mid \tilde{g}=\frac{1}{2} \partial_{\rho} \partial_{\sigma}\left(\mathcal{M}^{[\mu \rho][\sigma \nu]}+\mathcal{M}^{[\nu \rho][\sigma \mu]}\right)+B \sqrt{-\eta} \eta^{\mu \nu},
$$

где $\mathcal{M}^{\mu \rho \sigma \nu}$ - тензорная плотность веса 1 , а $B$ - константа. Этот результат следует из обратной леммы Пуанкаре в пространстве-времени Минковского. Тензоры $\mathcal{M}^{\mu \rho \sigma \nu}$ нельзя выбрать произвольным образом, но их необходимо выбрать так, чтобы обеспечить член $\delta S_{\mathrm{f}} / \delta \tilde{\gamma}_{\mu \nu}$. Поэтому свободу в добавлении произвольного ротора следует использовать вполне определенным образом. Как показал Хаггинс (аспирант Фейнмана) в своей диссертации [41], а Падманабан недавно снова подчеркнул [19], из-за наличия членов в виде такого ротора взаимодействие поля спина 2 с тензором энергии-импульса не ведет к единственной теории. Вместо этого, как полагает 
Хаггинс ([41], с. 3), “... необходимо дополнительное требование. Для Фейнмана это требование состояло в том, чтобы уравнения движения получались из принципа действия; Эйнштейн требовал, чтобы гравитационное поле имело геометрическую интерпретацию. Фейнман показал, что эти два требования эквивалентны."

Собирая все зависимые от $\eta_{\mu \nu}$ члены (с независимым $\tilde{g}_{\mu \nu}$ ) вместе, получаем $S=$ $S_{1}\left[\tilde{g}_{\mu \nu}, u\right]+S_{2}\left[\tilde{g}_{\mu \nu}, \eta_{\mu \nu}, u\right]$. Легко проверить, что если

$$
S_{2}=\frac{1}{2} \int d^{4} x R_{\mu \nu \rho \sigma}(\eta) \mathcal{M}^{\mu \nu \rho \sigma}\left(\eta_{\mu \nu}, \tilde{g}_{\mu \nu}, u\right)+\int d^{4} x \alpha^{\mu}{ }_{, \mu}+2 B \int d^{4} x \sqrt{-\eta},
$$

то $\delta S_{2} / \delta \eta_{\mu \nu} \mid \tilde{g}$ имеет в точности желаемый вид, а $S_{2}$ не влияет на уравнения ЭйлераЛагранжа, поскольку тождественно выполнены равенства $\delta S_{2} / \delta \tilde{g}_{\mu \nu}=0$ и $\delta S_{2} / \delta u=$ $0[4]^{6)}$. Коэффициент $B$ при члене, выражающем собой 4-объем, естественным образом выбирается так, чтобы сократить любой член нулевого порядка (например, обусловленный космологической постоянной) в действии, так чтобы действие обращалось в нуль в отсутствие гравитационного поля. Наличие 4-дивергенции $\alpha^{\mu}{ }_{, \mu}$ устраняет сомнения [19] по поводу получения членов, не аналитичных по постоянной взаимодействия $\lambda$. Не ясно, является ли гильбертово действие так или иначе наилучшим, если принять во внимание плохое поведение отвечающих ему законов сохранения [42].

Таким образом, действие с универсальным взаимодействием для ковариантной тензорной плотности имеет вид

$$
S=S_{1}\left[\tilde{g}_{\mu \nu}, u\right]+\frac{1}{2} \int d^{4} x R_{\mu \nu \rho \sigma}(\eta) \mathcal{M}^{\mu \nu \rho \sigma}+2 B \int d^{4} x \sqrt{-\eta}+\int d^{4} x \alpha^{\mu},{ }_{\mu} .
$$

Здесь мы имеем граничный член; если $\alpha^{\mu}-$ векторная плотность веса 1 , то $S-$ координатный скаляр. Используя эффективную искривленную плотность метрики $\tilde{g}_{\mu \nu}$, можно определить эффективную искривленную метрику как $\tilde{g}_{\mu \nu}=g_{\mu \nu}(\sqrt{-g})^{-l}$, а обратную искривленную плотность метрики - как $\tilde{g}^{\mu \nu}=g^{\mu \nu}(\sqrt{-g})^{l}$.

В качестве $S_{1}$ выберем гильбертово действие для общей теории относительности и минимально взаимодействующей материи с космологической постоянной:

$$
S_{1}=\frac{1}{16 \pi G} \int d^{4} x \sqrt{-g} R(g)-\frac{\Lambda}{8 \pi G} \int d^{4} x \sqrt{-g}+S_{\text {matter }}\left[g_{\mu \nu}, u\right] .
$$

Как хорошо известно, гильбертово действие - простейшее (скалярное) действие, которое можно построить, используя только метрический тензор. Если гравитационное поле всюду обращается в нуль, то гравитационное действие должно также обращаться в нуль. В безмассовом случае результат состоит в том, что $B=$ $\Lambda /(16 \pi G)$. Для обобщения на случай ненулевой массы, которое рассматривается

${ }^{6)}$ Если кажется, что использование члена $\frac{1}{2} \int d^{4} x R_{\mu \nu \rho \sigma}(\eta) \mathcal{M}^{\mu \nu \rho \sigma}\left(\eta_{\mu \nu}, \tilde{g}_{\mu \nu}, u\right)-$ слишком хитрое дело для того, чтобы до него можно было додуматься, не зная заранее теорию Эйнштейна, а потому происходит жульничание [19], то следует просто добавить к метрическому тензору энергии-импульса симметричный роторный член $\partial_{\rho} \partial_{\sigma}\left(\mathcal{M}^{[\mu \rho]}[\sigma \nu]+\mathcal{M}^{[\nu \rho][\sigma \mu]}\right) / 2+B \sqrt{-\eta} \eta^{\mu \nu}$, используя обычную недоопределенность тензора энергии-импульса. 
ниже, калибровочно-неинвариантная часть массового члена дает еще один вклад нулевого порядка, который также требует сокращения. При желании можно сделать так, чтобы материя взаимодействовала с тензором Римана для $g_{\mu \nu}$, или допустить появление высших степеней тензора Римана в гравитационном действии. В безмассовом случае можно положить $\Lambda=0$ [23].

\section{3. МАССИВНАЯ ГРАВИТАЦИЯ С УНИВЕРСАЛЬНЫМ ВЗАИМОДЕЙСТВИЕМ ДЛЯ ПОТЕНЦИАЛА В ВИДЕ КОВАРИАНТНОЙ ТЕНЗОРНОЙ ПЛОТНОСТИ}

Наша цель состоит в том, чтобы обобщить приведенный выше вывод таким образом, чтобы получить один или более вариантов массивных уравнений Эйнштейна в конечных пределах. Такие полевые уравнения связаны с уравнениями Эйнштейна подобно тому, как массивные уравнения Прока для электромагнитного поля соотносятся с уравнениями Максвелла. Однако массивная теория со спином 2 и спином 0 будет иметь духи на уровне свободной линейной теории. Вообще говоря, хорошее линейное поведение можно рассматривать как предпосылку для хорошего нелинейного поведения. Однако хорошее линейное поведение не кажется ни необходимым, ни достаточным для хорошего нелинейного поведения. В рассматриваемом случае представляется вполне возможным, что нелинейный вид гамильтоновых связей исправляет плохое поведение линейной теории. Это вкратце обсуждается ниже.

Можно ожидать, что массовый член для свободного поля является квадратичным по потенциалу и не содержит членов с производными. При этом предполагается, что действие свободного поля $S_{\mathrm{f}}$ имеет две части: часть $S_{\mathrm{fo}}$ (в основном кинетическую), инвариантную относительно бывших калибровочных преобразований, как и в рассмотренном выше безмассовом случае, и алгебраический массовый член $S_{\mathrm{fm}}$, являющийся квадратичным и нарушающий калибровочную симметрию. Мы ищем полную теорию с универсальным взаимодействием с действием $S$, которое имеет две составляющие: $S=S_{0}+S_{\mathrm{ms}}$. Они представляют собой знакомую часть $S_{0}$ (приводящую к тензору Эйнштейна, действию материи, космологической постоянной и 4-объемному члену нулевого порядка) и новую калибровочно-неинвариантную часть $S_{\mathrm{ms}}$, которая также имеет еще один 4-объемный член нулевого порядка. Как оказывается, массовый член построен как из алгебраической части действия $S_{0}$ (космологическая постоянная и 4-объемный член), так и из чисто алгебраического члена $S_{\mathrm{ms}}$.

Требование, чтобы при вариации $\tilde{\gamma}_{\mu \nu} \rightarrow \tilde{\gamma}_{\mu \nu}+\partial_{\mu} \tilde{\xi}_{\nu}+\partial_{\nu} \tilde{\xi}_{\mu}+c \eta_{\mu \nu} \partial^{\alpha} \tilde{\xi}_{\alpha}$ при $c \neq-1 / 2$ действие $S_{\mathrm{fo}}$ изменялось только на граничные члены, приводит к тождеству

$$
\partial_{\mu}\left(\frac{\delta S_{\mathrm{f} 0}}{\delta \tilde{\gamma}_{\mu \nu}}-\frac{l}{2} \eta^{\mu \nu} \eta_{\sigma \alpha} \frac{\delta S_{\mathrm{f} 0}}{\delta \tilde{\gamma}_{\sigma \alpha}}\right)=0 .
$$

Мы снова постулируем универсальное взаимодействие в виде

$$
\frac{\delta S}{\delta \tilde{\gamma}_{\mu \nu}}=\frac{\delta S_{\mathrm{f}}}{\delta \tilde{\gamma}_{\mu \nu}}-\lambda \frac{\delta S}{\delta \tilde{\eta}_{\mu \nu}} .
$$


Переход к биметрическим переменным $\tilde{g}_{\mu \nu}$ и $\tilde{\eta}_{\mu \nu}$, как и прежде, приводит к уравнению

$$
\frac{\delta S_{\mathrm{f}}}{\delta \tilde{\gamma}_{\mu \nu}}=\lambda \frac{\delta S}{\delta \tilde{\eta}_{\mu \nu}} \mid \tilde{g}
$$

Введем теперь величины $S_{\mathrm{fm}}$ и $S_{\mathrm{ms}}, S_{\mathrm{f}}=S_{\mathrm{f} 0}+S_{\mathrm{fm}}$ и $S=S_{0}+S_{\mathrm{ms}}$, чтобы рассматривать части, существующие в безмассовом случае, отдельно от дополнительных слагаемых в массивном случае. Таким образом, получаем уравнение

$$
\frac{\delta S_{\mathrm{f} 0}}{\delta \tilde{\gamma}_{\mu \nu}}+\frac{\delta S_{\mathrm{fm}}}{\delta \tilde{\gamma}_{\mu \nu}}=\lambda \frac{\delta S_{0}}{\delta \tilde{\eta}_{\mu \nu}}\left|\tilde{g}+\lambda \frac{\delta S_{\mathrm{ms}}}{\delta \tilde{\eta}_{\mu \nu}}\right| \tilde{g} .
$$

При допущении о соответствии новых членов $S_{\mathrm{fm}}$ и $S_{\mathrm{ms}}$ это уравнение разделяется на знакомую часть $\delta S_{\mathrm{fo}} / \delta \tilde{\gamma}_{\mu \nu}=\lambda\left(\delta S_{0} / \delta \tilde{\eta}_{\mu \nu}\right) \mid \tilde{g}$ и новую часть $\delta S_{\mathrm{fm}} / \delta \tilde{\gamma}_{\mu \nu}=$ $\lambda\left(\delta S_{\mathrm{ms}} / \delta \tilde{\eta}_{\mu \nu}\right) \mid \tilde{g}$. Из инвариантности (19) выводим, что $S_{0}$ имеет вид

$$
S_{0}=S_{1}\left[\tilde{g}_{\mu \nu}, u\right]+S_{2},
$$

как и в безмассовом случае. Снова выбираем простейший случай и получаем гильбертово действие с космологической постоянной, причем материя взаимодействует только с искривленной метрикой.

Новая часть в массивном случае имеет вид

$$
\frac{\delta S_{\mathrm{fm}}}{\delta \tilde{\gamma}_{\mu \nu}}=\lambda \frac{\delta S_{\mathrm{ms}}}{\delta \tilde{\eta}_{\mu \nu}} \mid \tilde{g} .
$$

В предположении, что свободно-полевой массовый член квадратичен по гравитационному потенциалу, его вариационная производная есть

$$
\frac{\delta S_{\mathrm{fm}}}{\delta \tilde{\gamma}_{\mu \nu}}=a \sqrt{-\eta} \tilde{\gamma}_{\alpha \beta}\left(\tilde{\eta}^{\alpha \mu} \tilde{\eta}^{\beta \nu}+b \tilde{\eta}^{\alpha \beta} \tilde{\eta}^{\mu \nu}\right) .
$$

Переход к биметрическим переменным дает

$$
\frac{a \sqrt{-\eta}}{\lambda}\left(-\tilde{g}_{\alpha \beta}+\tilde{\eta}_{\alpha \beta}\right)\left(\tilde{\eta}^{\alpha \mu} \tilde{\eta}^{\beta \nu}+b \tilde{\eta}^{\alpha \beta} \tilde{\eta}^{\mu \nu}\right)=\lambda \frac{\delta S_{\mathrm{ms}}}{\delta \tilde{\eta}_{\mu \nu}} \mid \tilde{g} .
$$

Возьмем выражение для $S_{\mathrm{ms}}$ в естественном виде:

$$
S_{\mathrm{ms}}=\int d^{4} x\left(p \tilde{g}_{\alpha \beta} \tilde{\eta}^{\alpha \beta}+q\right) \sqrt{-\eta},
$$

где $p$ и $q$ - некоторые вещественные числа, которые будут определены ниже. Заметим, что член $\sqrt{-g}$, дающий космологическую постоянную, сам по себе здесь не играет роли и уже включен в $S_{0}$. Используя соотношение $\sqrt{-\eta}=(\sqrt{-\tilde{\eta}})^{1 /(1-2 l)}$, вычисляем $\left(\delta S_{\mathrm{ms}} / \delta \tilde{\eta}_{\mu \nu}\right) \mid \tilde{g}$. Приравнивание $\lambda\left(\delta S_{\mathrm{ms}} / \delta \tilde{\eta}_{\mu \nu}\right) \mid \tilde{g}$ и $\delta S_{\mathrm{fm}} / \delta \tilde{\gamma}_{\mu \nu}$, т.е. приравнивание соответствующих коэффициентов, позволяет определить некоторые из постоянных. Приравнивание коэффициентов при членах $\sqrt{-\eta} \tilde{\eta}_{\mu \nu}$ дает $q=(2-4 l) a \times$ $(1+4 b) / \lambda^{2}$, коэффициентов при членах $\sqrt{-\eta} \tilde{\eta}^{\mu \nu} \tilde{\eta}^{\alpha \beta} \tilde{g}_{\alpha \beta}$ дает $p=-a b(2-4 l) / \lambda^{2}$, 
коэффициентов при членах $\sqrt{-\eta} \tilde{\eta}^{\alpha \mu} \tilde{\eta}^{\beta \nu} \tilde{g}_{\alpha \beta}$ дает $p=a / \lambda^{2}$. Совместное использование последних двух результатов дает $b=1 /(4 l-2)$, а все три результата дают $q=-2 a(2 l+1) / \lambda^{2}$. Комбинация алгебраической части действия $S_{0}$ с $S_{\mathrm{ms}}$ дает

$$
\begin{aligned}
S_{\text {alg }}=- & \frac{\Lambda}{8 \pi G} \int d^{4} x \sqrt{-g}+2 B \int d^{4} x \sqrt{-\eta}+ \\
& +\frac{a}{\lambda^{2}} \int d^{4} x\left(\tilde{g}_{\alpha \beta} \tilde{\eta}^{\alpha \beta}-4 l-2\right) \sqrt{-\eta} .
\end{aligned}
$$

Когда гравитационный потенциал обращается в нуль, величина $S_{\text {alg }}$ также должна обращаться в нуль, поэтому член нулевого порядка должен обращаться в нуль. Наложение этого условия дает

$$
B=\frac{\Lambda}{16 \pi G}-\frac{a(1-2 l)}{\lambda^{2}}
$$

Поскольку наша цель состоит в нахождении массивного обобщения теории Эйнштейна, а не некоторой теории с эффективной космологической постоянной, потребуем, чтобы член первого порядка в $\tilde{\gamma}_{\mu \nu}$ также обращался в нуль. Поскольку $\lambda^{2}=32 \pi G$, получаем $\Lambda=a(1-2 l) / 2$. Таким образом, знак члена с формальной космологической постоянной зависит от веса плотности исходно выбранного потенциала. Ожидается также, что квадратичная часть алгебраической составляющей действия $S_{\mathrm{alg}}$ будет согласована с массовым членом свободного поля $S_{\mathrm{fm}}$. После биномиального разложения и некоторых алгебраических преобразований становится очевидно, что именно это и имеет место ${ }^{7)}$. Разложение слабого поля для полного массивного нелинейного действия $S$ позволяет связать коэффициент $a$ с массой $m$ гравитонов спина 2: $a=-m^{2}$. Для нетахионных теорий требуется выполнение условия $a<0$.

Собранные вместе, эти результаты дают полное массивное действие $S$, которое зависит от массы $m$ гравитона спина 2 и параметра $l$, который управляет относительной массой духа спина 0:

$$
\begin{aligned}
S=\frac{1}{16 \pi G} \int d^{4} x \sqrt{-g} R(g)+S_{\text {matter }}\left[\tilde{g}_{\mu \nu}, u\right]+ \\
\quad+\frac{1}{2} \int d^{4} x R_{\mu \nu \rho \sigma}(\eta) \mathcal{M}^{\mu \nu \rho \sigma}\left[\tilde{\eta}_{\mu \nu}, \tilde{g}_{\mu \nu}, u\right]+\int d^{4} x \alpha^{\mu},_{\mu}- \\
\quad-\frac{m^{2}}{16 \pi G} \int d^{4} x\left[\sqrt{-g}(2 l-1)-\sqrt{-\eta}(2 l+1)+\frac{1}{2} \sqrt{-\eta} \tilde{g}_{\mu \nu} \tilde{\eta}^{\mu \nu}\right],
\end{aligned}
$$

где $l \neq 1 / 2$. Все эти теории являются теориями с универсальным взаимодействием, несмотря на то что в работах [23], [24] утверждается, что только теория ФМШ обладает этим свойством.

7) В работе [24] имеется ошибка в биномиальном разложении $\sqrt{-g}$ между уравнениями (43) и (44). 
Уравнения Эйлера-Лагранжа легко получить, если метрика $g_{\mu \nu}$ используется в качестве динамической переменной. Результат имеет вид

$$
\begin{aligned}
\frac{\delta S}{\delta g_{\mu \nu}}=- & \frac{\sqrt{-g}}{16 \pi G} G^{\mu \nu}-\frac{m^{2}}{16 \pi G}\left[\frac{2 l-1}{2} \sqrt{-g} g^{\mu \nu}+\right. \\
& \left.+\frac{(\sqrt{-\eta})^{l+1}}{4(\sqrt{-g})^{l}}\left(2 \eta^{\mu \nu}-l \eta^{\alpha \beta} g_{\alpha \beta} g^{\mu \nu}\right)\right]+\frac{\delta S_{\text {matter }}}{\delta g_{\mu \nu}} .
\end{aligned}
$$

Следуя Булверу и Дезеру [24], можно линеаризовать эти теории и проверить, действительно ли компонента спина 0 является тахионной. В нетахионном случае $-1 / 2 \leqslant l<1 / 2$. При $l=0$ дух спина 0 имеет ту же массу, что и степени свободы спина 2, так что эта теория (с весом плотности 0 для потенциала) является самой чистой среди всего их множества. (Связь между весом плотности и массой гравитона представляет собой занятное лингвистическое недоразумение.) Исследование различных масс для степени свободы спина 0 может привести к некоторым эмпирическим следствиям. Это происходит главным образом в однородных ситуациях при наличии большого масштаба, например в космологии [21]. Отношение массы $m_{0}$ спина 0 к массе $m_{2}$ спина 2 задается равенством

$$
\frac{m_{0}^{2}}{m_{2}^{2}}=\frac{-4 l^{2}+1}{2 l^{2}+1} .
$$

Таким образом, масса является четной функцией $l$. При $l=-1 / 2$ степень свободы спина 0 является безмассовой и множитель $\sqrt{-\eta}$ отсутствует в массовом члене. При $l \rightarrow 1 / 2$ скаляр снова становится легким и коэффициент при $\sqrt{-g}$ стремится к нулю, хотя значение $l=1 / 2$ запрещено. Между этими безмассовыми граничными точками степень свободы спина 0 становится тяжелее. В средней точке $l=0$ скаляр имеет ту же массу, что и поле спина 2, что придает простой вид волновому уравнению для линеаризованных массивных уравнений Эйнштейна. Таким образом, дух спина 0 никогда не оказывается тяжелее, чем степени свободы спина 2 , так что теории с $l \neq 0$ имеют более слабое гравитационное притяжение на больших расстояниях и в однородных ситуациях.

\section{4. ВЫВОД УРАВНЕНИЙ ДЛЯ КОНТРАВАРИАНТНОЙ ТЕНЗОРНОЙ ПЛОТНОСТИ ПОТЕНЦИАЛА: БЕЗМАССОВЫЙ СЛУЧАЙ}

Кратко представим контравариантный аналог приведенного выше вывода уравнений Эйнштейна. Гравитационный потенциал при этом является полем контравариантной симметричной тензорной плотности $\tilde{\gamma}^{\mu \nu}$ с весом плотности $l, l \neq 1 / 2$. Помимо очевидного перемещения некоторых индексов, введем в контравариантном случае некоторые различия в знаках. Надежда на простое правило, связывающее перемещение индексов и изменения в знаках, разрушается, поскольку симметрия между $(0,2)$-теорией веса $-l$ и $(2,0)$-теорией веса $l$ нарушена: плотность лагранжиана является скалярной плотностью веса 1 , а не веса 0 . Следствия этой несовершенной 
симметрии в рассматриваемом ниже массивном случае проявляются сильнее, чем в безмассовом. Будет удобно использовать не саму обратную плоскую метрику, а близкую ей, вес плотности $l$ вида $\tilde{\eta}^{\mu \nu}=\eta^{\mu \nu}(\sqrt{-\eta})^{l}$, при $l \neq 1 / 2$.

В безмассовых теориях предполагается исходная инфинитезимальная инвариантность (с точностью до граничного члена) свободного гравитационного действия $S_{\mathrm{f}}$ относительно инфинитезимальных калибровочных преобразований $\tilde{\gamma}^{\mu \nu} \rightarrow \tilde{\gamma}_{\mu \nu}+$ $\delta \tilde{\gamma}^{\mu \nu}$, где

$$
\delta \tilde{\gamma}^{\mu \nu}=\partial^{\mu} \tilde{\xi}^{\nu}+\partial^{\nu} \tilde{\xi}^{\mu}+c \eta^{\mu \nu} \partial_{\alpha} \tilde{\xi}^{\alpha}
$$

$c \neq-1 / 2, \tilde{\xi}^{\nu}-$ произвольная векторная плотность веса $l$. Оказывается целесообразным положить $c=-l$.

Для любого $S_{\mathrm{f}}$, инвариантного в этом смысле, дивергенция некоторой линейной комбинации свободно-полевых уравнений тождественно равна нулю:

$$
\partial^{\mu}\left(\frac{\delta S_{\mathrm{f}}}{\delta \tilde{\gamma}^{\mu \nu}}-\frac{l}{2} \eta_{\mu \nu} \eta^{\sigma \alpha} \frac{\delta S_{\mathrm{f}}}{\delta \tilde{\gamma}^{\sigma \alpha}}\right)=0 .
$$

Это обобщенное тождество Бьянки для свободно-полевой теории. Локальное сохранение энергии-импульса, которое имеет место при использовании уравнений ЭйлераЛагранжа для гравитации $\tilde{\gamma}^{\mu \nu}$ и материи $u$, можно записать как

$$
\partial^{\nu}\left(\frac{\delta S}{\delta \tilde{\eta}^{\mu \nu}}-\frac{l}{2} \tilde{\eta}^{\alpha \beta} \tilde{\eta}_{\mu \nu} \frac{\delta S}{\delta \tilde{\eta}_{\alpha \beta}}\right)=0 .
$$

Постулат универсального взаимодействия запишем в виде

$$
\frac{\delta S}{\delta \tilde{\gamma}^{\mu \nu}}=\frac{\delta S_{\mathrm{f}}}{\delta \tilde{\gamma}^{\mu \nu}}+\lambda \frac{\delta S}{\delta \tilde{\eta}^{\mu \nu}}
$$

(причина выбора знака коэффициента перед членом, включающим тензор энергииимпульса, вскоре станет ясной). Мы получаем величину $S$, переходя от переменных $\tilde{\gamma}^{\mu \nu}$ и $\tilde{\eta}^{\mu \nu}$ к биметрическим переменным $\tilde{g}^{\mu \nu}$ и $\tilde{\eta}^{\mu \nu}$, где

$$
\tilde{g}^{\mu \nu}=\tilde{\eta}^{\mu \nu}+\lambda \tilde{\gamma}^{\mu \nu}
$$

Коэффициент при $\lambda \tilde{\gamma}^{\mu \nu}$ был выбран так, чтобы в той мере, в какой это легко сделать, ковариантный и контравариантный случаи определяли гравитационный потенциал аналогичным образом. В частности, в линейном порядке по потенциалу при использовании декартовых координат бесследовая часть гравитационного потенциала $\gamma$ имеет одну и ту же значимость с точки зрения наблюдений, независимо от того, является она разностью между ковариантной искривленной и плоской и искривленной плотностями метрики или разностью между контравариантной плоской и 
искривленной плотностями метрики ${ }^{8)}$. Приравнивая коэффициенты при вариациях, получаем

$$
\frac{\delta S}{\delta \tilde{\eta}^{\mu \nu}}\left|\tilde{\gamma}=\frac{\delta S}{\delta \tilde{\eta}^{\mu \nu}}\right| \tilde{g}+\frac{\delta S}{\delta \tilde{g}^{\mu \nu}}
$$

и

$$
\frac{\delta S}{\delta \tilde{\gamma}^{\mu \nu}}=\lambda \frac{\delta S}{\delta \tilde{g}^{\mu \nu}},
$$

откуда получаем

$$
\frac{\delta S_{\mathrm{f}}}{\delta \tilde{\gamma}^{\mu \nu}}=-\lambda \frac{\delta S}{\delta \tilde{\eta}^{\mu \nu}} \mid \tilde{g} .
$$

Использование обобщенного тождества Бьянки дает разбиение $S$ на составляющую $S_{1}\left[\tilde{g}^{\mu \nu}, u\right]$ и часть $S_{2}$, которая принимает вид (16). Величина $S_{2}$ содержит всю неустранимую зависимость от фоновой метрики и не дает вклада в полевые уравнения. Простейший выбор для $S_{1}$ дает гильбертово действие для уравнений Эйнштейна, а также космологическую постоянную, как и в ковариантном случае. В безмассовом случае конкретный выбор из разрешенных значений $l$ не играет роли.

\section{5. ВЫВОД УРАВНЕНИЙ ДЛЯ КОНТРАВАРИАНТНОЙ ТЕНЗОРНОЙ ПЛОТНОСТИ ПОТЕНЦИАЛА: МАССИВНЫЙ СЛУЧАЙ}

Для обобщения приведенного вывода уравнений для контравариантной тензорной плотности на массивный случай, как и в ковариантном случае, имеет значение выбор веса плотности $l$. Теория ФМШ-Логунова оказывается контравариантной массивной теорией с универсальным взаимодействием веса $l=1$. Хотя с некоторых определенных точек зрения теория веса $l=1$ - наилучшая среди контравариантных массивных теорий, она не является единственной такой теорией. Действие свободного поля $S_{\mathrm{f}}$ по-прежнему состоит из двух частей - части $S_{\mathrm{f} 0}$, являющейся преимущественно кинетической и имеющей локальную калибровочную симметрию, и алгебраического массового члена $S_{\mathrm{fm}}$. По-прежнему, действие полной теории $S$ разбивается на две части, $S_{0}$ и $S_{\mathrm{ms}}$.

Требование, чтобы $S_{\mathrm{fo}}$ изменялось только на граничный член при инфинитезимальных вариациях,

$$
\delta \tilde{\gamma}^{\mu \nu}=\partial^{\mu} \tilde{\xi}^{\nu}+\partial^{\nu} \tilde{\xi}^{\mu}-l \eta^{\mu \nu} \partial_{\alpha} \tilde{\xi}^{\alpha}
$$

\footnotetext{
${ }^{8)}$ Если $g_{\mu \nu}=\eta_{\mu \nu}-\lambda \gamma_{\mu \nu}$, то обратная метрика приводит (по крайней мере, формально) к разложению в бесконечный ряд, первый член которого имеет знак, отличный от предлагаемого наивным поднятием индексов: $g_{\mu \nu}=\eta_{\mu \nu}+\lambda \gamma_{\mu \nu}+\cdots$, откуда следует, что $g^{\mu \nu}-\eta^{\mu \nu} \approx \lambda \gamma^{\mu \nu}$, а не $-\lambda \gamma_{\mu \nu}$. Если выполнено точное соотношение $g^{\mu \nu}-\eta^{\mu \nu}=\lambda \psi^{\mu \nu}$ (знак у $\lambda$-члена важен), то $\psi_{\mu \nu} \approx \gamma_{\mu \nu}$. Таким образом, смысл гравитационного потенциала не чувствителен к изменению знака, так что сравнивать различные массивные теории становится легче.
} 
при $l \neq 1 / 2$ приводит к тождеству

$$
\partial^{\mu}\left(\frac{\delta S_{\mathrm{f} 0}}{\delta \tilde{\gamma}^{\mu \nu}}-\frac{l}{2} \eta_{\mu \nu} \eta^{\sigma \alpha} \frac{\delta S_{\mathrm{f0}}}{\delta \tilde{\gamma}^{\sigma \alpha}}\right)=0 .
$$

Мы снова постулируем универсальное взаимодействие в виде (33). Перейдем к биметрическим переменным $\tilde{g}^{\mu \nu}$ и $\tilde{\eta}^{\mu \nu}$. Добиваясь, чтобы новые массовые члены и члены, присутствовавшие ранее, были согласованными по отдельности, находим, что массовые члены подчиняются уравнению

$$
\frac{\delta S_{\mathrm{fm}}}{\delta \tilde{\gamma}^{\mu \nu}}=-\lambda \frac{\delta S_{\mathrm{ms}}}{\delta \tilde{\eta}^{\mu \nu}} \mid \tilde{g} .
$$

Действие $S_{\mathrm{fm}}$ выбирается квадратичным по гравитационному потенциалу и таким, чтобы было выполнено соотношение

$$
\frac{\delta S_{\mathrm{fm}}}{\delta \tilde{\gamma}^{\mu \nu}}=a \sqrt{-\eta} \tilde{\gamma}^{\alpha \beta}\left(\tilde{\eta}_{\alpha \mu} \tilde{\eta}_{\beta \nu}+b \tilde{\eta}_{\alpha \beta} \tilde{\eta}_{\mu \nu}\right) .
$$

Величину $S_{\mathrm{ms}}$ естественно выбрать в виде

$$
S_{\mathrm{ms}}=\int d^{4} x\left(p \tilde{g}^{\alpha \beta} \tilde{\eta}_{\alpha \beta}+q\right) \sqrt{-\eta}
$$

с нефиксированными $p$ и $q$. Сравнение коэффициентов при нескольких членах дает

$$
\begin{aligned}
q & =\frac{-(2-4 l) a(1+4 b)}{\lambda^{2}}, & p & =\frac{a b(2-4 l)}{\lambda^{2}}, \quad p=\frac{a}{\lambda^{2}}, \\
b & =-\frac{1}{4 l-2}, & q & =\frac{2 a(2 l-3)}{\lambda^{2}} .
\end{aligned}
$$

Требование, чтобы алгебраический член нулевого порядка в $S$ обращался в нуль, дает

$$
B=\frac{\Lambda}{16 \pi G}+\frac{a(1-2 l)}{\lambda^{2}} .
$$

А требование, чтобы алгебраический член первого порядка обращался в нуль, после некоторых алгебраических преобразований дает $\Lambda=-a(1-2 l) / 2$. Как можно было надеяться, член второго порядка согласован с массовым членом свободного поля. Масса $m$ гравитона спина 2 определяется как $a=-m^{2}$.

Собирая все эти результаты, получаем полное массивное действие $S$, которое зависит от массы $m$ гравитона спина 2 и параметра $l$, который управляет относительной массой гравитона спина 0:

$$
\begin{aligned}
S= & \frac{1}{16 \pi G} \int d^{4} x \sqrt{-g} R(g)+S_{\text {matter }}\left[\tilde{g}^{\mu \nu}, u\right]+ \\
& +\frac{1}{2} \int d^{4} x R_{\mu \nu \rho \sigma}(\eta) \mathcal{M}^{\mu \nu \rho \sigma}\left[\tilde{\eta}^{\mu \nu}, \tilde{g}^{\mu \nu}, u\right]+\int d^{4} x \alpha^{\mu},_{\mu}- \\
& -\frac{m^{2}}{16 \pi G} \int d^{4} x\left[-\sqrt{-g}(2 l-1)+\sqrt{-\eta}(2 l-3)+\frac{1}{2} \sqrt{-\eta} \tilde{g}^{\mu \nu} \tilde{\eta}_{\mu \nu}\right],
\end{aligned}
$$


где $l \neq 1 / 2$. Среди теорий с универсальным взаимодействием, рассматриваемых в данной статье, отсутствует эмпирически сомнительный массовый член ФирцаПаули. Все эти теории содержатся в двухпараметрическом семействе теорий Огиевецкого и Полубаринова.

Хотя для нахождения уравнений Эйлера-Лагранжа можно было бы использовать контравариантный тензор (или тензорную плотность), эти уравнения легче сравнивать с найденными ранее, если используется метрика $g_{\mu \nu}$. Получающиеся уравнения имеют вид

$$
\begin{aligned}
\frac{\delta S}{\delta g_{\mu \nu}}=- & \frac{\sqrt{-g}}{16 \pi G} G^{\mu \nu}-\frac{m^{2}}{16 \pi G}\left[\frac{1-2 l}{2} \sqrt{-g} g^{\mu \nu}+\right. \\
& \left.+\frac{(\sqrt{-g})^{l} \eta_{\alpha \beta}}{4(\sqrt{-\eta})^{l-1}}\left(l g^{\alpha \beta} g^{\mu \nu}-2 g^{\mu \alpha} g^{\nu \beta}\right)\right]+\frac{\delta S_{\text {matter }}}{\delta g_{\mu \nu}} .
\end{aligned}
$$

Линеаризация этих теорий показывает, что поле спина 0 не является тахионом тогда и только тогда, когда $1 / 2<l \leqslant 3 / 2$. При $l=1$ дух спина 0 имеет ту же массу, что и степени свободы спина 2 , так что эти полевые уравнения, отвечающие теории ФМШ-Логунова, являются самыми чистыми из всего семейства теорий, содержащих примесь духов спина 0 . В линейном приближении $(0,2)$-теория веса $-l$ тождественна $(2,0)$-теории веса $l+1$. Таким образом, для $(2,0)$-теории веса $l+1$ отношение масс гравитонов спина 0 и спина 2 равно

$$
\frac{m_{0}^{2}}{m_{2}^{2}}=\frac{-4(l-1)^{2}+1}{2(l-1)^{2}+1}
$$

Таким образом, масса является четной функцией $l-1$. При $l=3 / 2$ степень свободы спина 0 является безмассовой и в массовый член не входит $\sqrt{-\eta}$. При $l \rightarrow 1 / 2$ скаляр снова становится легким и коэффициент $\sqrt{-g}$ стремится к нулю, хотя значение $l=1 / 2$ запрещено. Между этими безмассовыми граничными точками степень свободы спина 0 становится тяжелее. В срединной точке $l=1$ (теория ФМШ-Логунова) гравитон спина 0 имеет ту же массу, что и гравитон спина 2 , что придает простой вид волновому уравнению для линеаризованных массивных уравнений Эйнштейна. Таким образом, дух спина 0 никогда не делается тяжелее, чем степени свободы спина 2 для этого семейства теорий, хотя так может случиться для большего семейства массивных теорий Огиевецкого и Полубаринова $[7]^{9)}$.

\section{6. МАССИВНЫЕ ГРАВИТАЦИИ И ЭКСПЕРИМЕНТ}

Как в экспериментальном [43], так и в теоретическом контекстах принято говорить о единственной массе единственного гравитона, как если бы все гравитоны имели одну и ту же массу. Хотя все гравитоны действительно имеют одну и ту же массу в наиболее известной теории массивной гравитации спина 2 и спина 0 ,

\footnotetext{
9) Весьма отрадно, что в специальном конформно-плоском случае $g_{\mu \nu}=\phi \eta_{\mu \nu}(2,0)$-теория веса
} $l+1$ и $(0,2)$-теория веса $-l$ совпадают. 
разработанной Френдом, Махешвари и Шонбергом и изучавшейся Логуновым с сотрудниками, существование теорий Огиевецкого и Полубаринова показывает, что массивная гравитация имеет два массивных параметра, которые должны определяться экспериментально. Желательно было бы выяснить, до какой степени молчаливое допущение равных масс гравитонов спина 2 и спина 0 в действительности используется при нахождении экспериментальных ограничений на массивную гравитацию. Астрофизические проверки изменения поведения тех степеней свободы, которые представлены в безмассовой общей теории относительности, прежде всего налагают ограничения на массу гравитона спина 2. Эмпирические ограничения на массу гравитона спина 2 столь жесткие, что часть массового члена, относящаяся к спину 2, эмпирически пренебрежима, за исключением случаев сильных полей или космических расстояний [20], [21]; эти режимы также изучались Логуновым и его сотрудниками. Гибкость в отношении массы гравитона спина 0 в массивных теориях, полученных в настоящей работе, открывает некоторые феноменологические возможности путем увеличения области отталкивания для спина 0, уравновешивающего некоторую часть притяжения за счет спина 2. Более широкое семейство массивных теорий Огиевецкого и Полубаринова позволяет иметь как бо́льшую, так и меньшую область отталкивания для спина 0 по сравнению с областью притяжения для спина 2. Подобную феноменологическую гибкость исследовали недавно Бабак и Грищук [21]. Они заметили, что их специальный случай массивного спина 2 и безмассового спина 0 согласуется с общей теорией относительности в космологическом контексте из-за высокой степени симметрии. Таким образом, космологические пределы на массы гравитонов главным образом связаны с массой спина 0. Хотя теории Огиевецкого и Полубаринова имеют нелинейные массовые члены, полученные из первых принципов, в отличие от линейных массовых членов Бабака и Грищука, мотивированных математической простотой, подобное же качественное поведение двух видов двухпараметрических массивных гравитаций можно ожидать и за пределами сильно нелинейных режимов.

В свете жестких эмпирических ограничений на массы гравитонов наблюдаемые следствия наличия массовых членов достаточно сложно детектировать. Имеются, однако, два важных теоретических вопроса, возникающих в классической теории для массивных вариантов уравнений Эйнштейна. Первый вопрос - это хорошо известный вопрос об устойчивости, положительности энергии и т.п. при наличии компоненты спина 0 с неправильным знаком. Второй вопрос касается причинности: массивная гравитация является теорией поля в рамках специальной теории относительности с наблюдаемой $\eta_{\mu \nu}$, но есть причины опасаться, что распространение поля может нарушать причинность за счет того, что световой конус эффективной метрики $g_{\mu \nu}$ вытекает наружу конуса для $\eta_{\mu \nu}$. K этим вопросам мы сейчас и обратимся. 


\section{7. ПОЛОЖИТЕЛЬНОСТЬ ЭНЕРГИИ}

Давно было показано [24], [44], что массивные варианты уравнений Эйнштейна приводят к неприятной дилемме: или массовый член имеет вид члена Фирца-Паули с $5 \infty^{3}$ степенями свободы (чистый спин 2) и эмпирически отвергается из-за отсутствия непрерывности в безмассовом пределе, или теория имеет $6 \infty^{3}$ степеней свободы, включая случай спина 0 с неправильным знаком (пространственная скалярная плотность), и в этом случае после линеаризации возникает неустойчивость. Указанная проблема - это проблема отсутствия непрерывности ван Дама-ВельтманаЗахарова [45], по которой в последние несколько лет, после долгого периода относительного затишья, появилась обширная литература. Более актуален для нас следующий вопрос: являются ли массивные теории с $6 \infty^{3}$ степенями свободы (спин 2 и спин 0) неустойчивыми?

Вопреки широко распространенным представлениям Виссер [20] привел аргументы в пользу того, что массивные теории с $6 \infty^{3}$ степенями свободы вполне могут быть устойчивыми. После этого Бабак и Грищук доказывали [21], что такие теории в действительности устойчивы. Что касается конкретного случая теории ФМШ, то сами авторы этой теории не были убеждены в неустойчивости [23], хотя они перестали заниматься этим вопросом после того, как были опубликованы аргументы в пользу неустойчивости. В середине и в конце 1980-х годов Логунов и его сотрудники (такие как Лоскутов и Чугреев) приняли теорию ФМШ в качестве массивного варианта релятивистской теории гравитации. Они утверждали [11], что эта конкретная теория вполне может быть устойчивой, несмотря на аргумент о линеаризации. Более убедительным является то, что Лоскутов [46] вычислил гравитационное излучение от ограниченного источника и пришел к выводу, что оно в действительности положительно определено, хотя теория и имеет компоненту спина 0 с неправильным знаком. Удивительно, что это заключение получило столь незначительный отклик.

Хотя вопрос о положительности энергии (или о положительности массы, как принято говорить в гравитационном контексте) не получил своего позитивного решения (в смысле общего доказательства, что все точные решения, подчиняющиеся некоторым энергетическим условиям, имеют положительную массу), равным образом не кажутся окончательно убедительными основанные на линеаризации аргументы в пользу неустойчивости. Используя гамильтонов формализм, полезно указать, что процедура линеаризации не вызывает доверия, поскольку она существенно изменяет вид гамильтоновых связей таким образом, что неустойчивость делается более вероятной, чем это имеет место в действительности для точной нелинейной теории. Хотя компонента спина 0 с неправильным знаком и присутствует в нелинейной теории, ее кинетическая энергия связана с кинетической энергией степеней свободы с правильным знаком таким образом, что она может легко излучаться только согласованно с излучением компонент со степенями свободы с положительной энергией. Именно это свойство теряется при линеаризации. Это важное свойство гамильтоновой свя- 
зи существенно зависит от члена кубичного порядка в плотности гамильтониана, а следовательно, квадратичного в полевых уравнениях.

В случае контравариантной теории ФМШ-Логунова веса 1 уравнения поля накладывают нижнее ограничение на плотность гамильтониана чуть ниже нуля, в отличие от граничных членов (которые должны уничтожаться, по крайней мере в статических случаях, из-за экспоненциального юкавского убывания гравитационного потенциала ограниченных систем). Пренебрегая несущественными членами и множителями из действия (43) при $l=1$, получаем плотность лагранжиана

$$
\mathcal{L}=\sqrt{-g} R(g)-m^{2}\left(-\sqrt{-g}-\sqrt{-\eta}+\frac{1}{2} \sqrt{-g} g^{\mu \nu} \eta_{\mu \nu}\right) .
$$

Используем $(3+1)$-мерное АДМ-разложение $\left.{ }^{10}\right)$ [32] и выберем координаты (декартовы, сферические и т.п.) так, чтобы $\eta_{00}=-1$ и $\eta_{0 i}=0$. Искривленная метрика $g_{\mu \nu}$ тем самым выражается в терминах функции хода $N$, связывающей эффективное собственное время с координатным временем, вектора сдвига $\beta^{i}$, отражающего, как пространственная система координат представляется сдвинутой по различным временнь́м срезам, и искривленной пространственной метрики $h_{i j}$, обратная к которой есть $h^{i j}$, а ее определитель равен $h$. Обозначая, как обычно, через $g^{\mu \nu}$ обратную искривленную метрику, имеем $g^{00}=-N^{-2}$ (здесь наиболее удобна обратная метрика), $g_{i j}=h_{i j}$ и $g_{0 i}=h_{i j} \beta^{j}$. Индексы трехмерных величин поднимаются и опускаются с помощью $h_{i j}$. Отбрасывая дивергенцию из указанного выше действия гильбертова типа, имеем массивный вариант ФМШ стандартной $(3+1)$-мерной плотности лагранжиана:

$$
\begin{aligned}
\mathcal{L}=N & \sqrt{h}\left[{ }^{3} R+K_{a b} K^{a b}-K^{2}+m^{2}\left(1-\frac{h^{i j} \eta_{i j}}{2}\right)\right]+ \\
& +m^{2}\left[\sqrt{-\eta}+\frac{\sqrt{h}}{2 N}\left(\eta_{i j} \beta^{i} \beta^{j}-1\right)\right]
\end{aligned}
$$

Всюду далее мы опускаем верхний индекс у ${ }^{3} R$.

Определяя канонические импульсы обычным образом, получаем обычные результаты:

$$
\pi^{i j}=\frac{\partial \mathcal{L}}{\partial h_{i j, 0}}=\sqrt{h}\left(K^{i j}-h^{i j} K\right), \quad P_{i}=\frac{\partial \mathcal{L}}{\partial \beta_{, 0}^{i}}=0, \quad P=\frac{\partial \mathcal{L}}{\partial N_{, 0}}=0 .
$$

Четыре обращающихся в нуль канонических импульса в контексте динамических систем со связями [47] называются первичными связями.

Выполняя обобщенное преобразование Лежандра и используя первичные связи, получаем каноническую плотность гамильтониана

$$
\mathcal{H}=N\left[\mathcal{H}_{0}+m^{2} \sqrt{h}\left(\frac{1}{2} h^{i j} \eta_{i j}-1\right)\right]+\beta^{i} \mathcal{H}_{i}-m^{2} \sqrt{-\eta}+\frac{m^{2} \sqrt{h}}{2 N}\left(1-\eta_{i j} \beta^{i} \beta^{j}\right),
$$

10) АДМ-разложение - нековариантное $(3+1)$-представление метрического тензора риманова пространства, впервые предложенное Арновиттом, Дезером и Мизнером. 
где, как обычно,

$$
\mathcal{H}_{0}=\frac{1}{\sqrt{h}}\left(\pi^{i j} \pi_{i j}-\frac{1}{2} \pi^{2}\right)-\sqrt{h} R, \quad \mathcal{H}_{i}=-2 D_{j} \pi_{i}^{j},
$$

а $D_{j}$ - трехмерная ковариантная производная без кручения, согласованная с $h_{i j}$. При $m=0$ воспроизводится обычный вид выражения, представляющего собой точно сумму связей, но при $m \neq 0$ этот вид нарушается, что приводит к шести, а не к двум степеням свободы. Заметим, что мы удержали член нулевого порядка $-m^{2} \sqrt{-\eta}$, так что пространство-время Минковского имеет нулевую энергию, как и должно быть (этот член был опущен Булвером и Дезером [24]). Варьируя функцию хода $N$ и вектор сдвига $\beta^{i}$, получаем вторичные связи, а именно модифицированные гамильтоновы связи

$$
\frac{\partial \mathcal{H}}{\partial N}=\mathcal{H}_{0}+m^{2} \sqrt{h}\left(-1+\frac{1}{2} h^{i j} \eta_{i j}\right)-\frac{m^{2} \sqrt{h}}{2 N^{2}}\left(1-\eta_{i j} \beta^{i} \beta^{j}\right)=0
$$

и модифицированные импульсные связи

$$
\frac{\partial \mathcal{H}}{\partial \beta^{i}}=\mathcal{H}_{i}-\frac{m^{2} \sqrt{h}}{N} \eta_{i j} \beta^{j}=0 .
$$

Это связи второго рода [48]. Как указывали Булвер и Дезер, эти соотношения можно использовать, чтобы исключить ход и сдвиг из плотности гамильтониана и выразить плотность гамильтониана на части массовой оболочки только через истинные степени свободы и их импульсы:

$$
\mathcal{H}=\sqrt{2 m^{2} \sqrt{h}\left[\mathcal{H}_{0}+m^{2} \sqrt{h}\left(\frac{h^{i j} \eta_{i j}}{2}-1\right)\right]+\mathcal{H}_{i} \mathcal{H}_{j} \eta^{i j}}-m^{2} \sqrt{-\eta} .
$$

Выражая ход в терминах истинных степеней свободы, получаем

$$
N^{2}=\frac{h m^{4}}{2 m^{2} \sqrt{h}\left[\mathcal{H}_{0}+m^{2} \sqrt{h}\left(h^{i j} \eta_{i j} / 2-1\right)\right]+\mathcal{H}_{i} \mathcal{H}_{j} \eta^{i j}} .
$$

Замена переменных от хода $N$ к популярной в последнее время "срезовой плотности" $\alpha=N / \sqrt{h}[49]$ позволяет записать плотность гамильтониана на массовой оболочке как $\mathcal{H}=m^{2}(1 / \alpha-\sqrt{-\eta})$. Граничные члены здесь опущены, но в некоторых случаях они обращаются в нуль из-за юкавского убывания для локализованных источников.

Рассмотрим теперь линеаризацию точной плотности гамильтониана. Достоинство срезовой плотности $\alpha=N / \sqrt{h}$ заключается в том, что она уменышает число радикалов в плотности гамильтониана вне массовой оболочки, а плотность гамильтониана на массовой оболочке принимает простой вид. До линеаризации мы имели

$$
\begin{aligned}
\mathcal{H}=\alpha & {\left[\pi^{i j} \pi^{k l}\left(h_{i k} h_{j l}-\frac{1}{2} h_{i j} h_{k l}\right)-h R+m^{2} h\left(-1+\frac{1}{2} h^{i j} \eta_{i j}\right)\right]+} \\
& +\beta^{i} \mathcal{H}_{i}-m^{2} \sqrt{-\eta}+\frac{m^{2}}{2 \alpha}\left(1-\eta_{i j} \beta^{i} \beta^{j}\right) .
\end{aligned}
$$


Пусть теперь $\eta_{i j}=\delta_{i j}, h_{i j}=\delta_{i j}+\phi_{i j}$ и $\alpha=1+a$. Нас интересует только кинетический член. Членами пятой и четвертой степени, содержащими $a \Pi^{2} \phi^{2}, \Pi^{2} \phi^{2}$ и $a \Pi^{2} \phi$ (индексы опущены), можно пренебречь, но при отбрасывании кубичного члена $a\left(\pi^{i j} \pi^{i j}-\pi^{i i} \pi^{j j} / 2\right)$ возникают серьезные трудности, связанные с членом $-\pi^{i i} \pi^{j j} / 2$, которых не было в точной теории. Кинетический член, содержащий беспокоящий нас скалярный вклад $-\pi^{2} / 2$ с неправильным знаком, находится в гамильтоновых связях точной теории, где связанные с ним сложности несколько смягчены, а после линеаризации член $-\pi^{2} / 2$ может приводить к таким проблемам, как излучение произвольно большой отрицательной энергии или возможность излучения с произвольно большой положительной энергией, что приводит к неустойчивости. Если бы имелись кубичные члены, такие как $a\left(\pi^{i j} \pi^{i j}-\pi^{i i} \pi^{j j} / 2\right)$ и, возможно, его аналог с пространственной производной, то приближенная гамильтонова связь по-прежнему существенно напоминала бы точный вид и не создавала бы серьезных трудностей.

Булвер и Дезер [24], обращая внимание на приведенное выше выражение для гамильтониана $\mathcal{H}$ на массовой оболочке, имеющее вид квадратного корня (и без члена нулевого порядка), отмечают, что “гамильтонов вид (в терминах шести степеней свободы) ... представляется положительно определенным. Поскольку, кроме того, рассматриваемое линеаризованное приближение соответствует скалярно-духовой примеси и тем самым дает линеаризованное взаимодействие Эйнштейна в пределе слабого поля, могло бы показаться, что данная модель имеет по крайней мере два усовершенствования по сравнению с .. [эмпирически сомнительной теорией Фирца-Паули и ее обобщениями]: ее энергия положительна и она имеет правильное линеаризованное поведение. Однако она неприемлема: вакуум является не локальным минимумом, а только седловой точкой, что можно заметить, рассматривая равновесные (статические) конфигурации или просто разлагая $H$ до квадратичного порядка, где обнаруживается, что он согласуется с линеаризованной (духовой) версией $H$. Тот факт, что для соответствующих возбуждений квадратичная часть H может быть отрицательной, может показаться несущественным ввиду явной положительности полного $H$. . . K сожалению, выражение под знаком квадратного корня не является существенно положительным .., хотя его положительность и требуется для того, чтобы теория имела смысл, т.е. для положительности $N^{2} \ldots$ (в противном случае эффективная риманова метрика, которую “видит" материя, становится патологической). Поэтому приходится наложить условие, чтобы возбуждения удовлетворяли этому требованию, т.е. произвольным образом отрезать те моды, которые уводят $H$ ниже его нулевого значения в вакуумном $(g=\eta)$ состоянии. Неустойчивость вблизи вакуума $(g \simeq \eta)$ является причиной для того, чтобы отвергнуть эту и другие модели, в которых квадратичная масса не имеет вида Паули-Фирца."

Такое рассуждение несколько озадачивает, поскольку случай $N \leqslant 0$ приводит к сингулярности. Таким образом, нет необходимости искусственно налагать условие $N>0$. Остается вопрос о том, настолько часто в теории реализуется случай $N=0$, чтобы сингулярности сформировались в реальной ситуации. Никаких аргументов 
на этот счет приведено не было, тогда как тот факт, что из стремления $N$ к нулю следует гравитационное замедление времени, указывает на то, что величина $N$ не склонна обращаться в нуль [50]. До тех пор пока $H=\int \mathcal{H} d^{3} x$ положительно (или неотрицательно) для некоторых подходящих граничных условий, нет необходимости для $\mathcal{H}$ быть всюду положительным. Восстановление отрицательного члена нулевого порядка в $\mathcal{H}$ означает, что гамильтониан $\mathcal{H}$ ограничен снизу, но не нулем.

В настоящий момент в теории ФМШ-Логунова или любой другой “духовой” теории из рассматриваемых здесь семейств, насколько нам известно, нет решений нелинейных полевых уравнений, точных или численных, которые имели бы отрицательную полную энергию. Аналогичная ситуация имеет место в отношении решений, указывающих на неустойчивость тем фактом, что они излучают отрицательную полную энергию. С учетом потребности в непертурбативном рассмотрении вопрос об устойчивости мог бы быть наилучшим образом решен с помощью релятивистов, занимающихся численными расчетами. Достаточно работать в рамках сферической симметрии, где поле неправильного знака может излучать, а большинство из полей с правильным знаком не могут.

\section{8. ПРИЧИННОСТЬ}

Поскольку массивные теории гравитации рассматриваются в пространстве-времени Минковского с наблюдаемой фоновой метрикой $\eta_{\mu \nu}$, заслуживает внимания вопрос о том, является ли нулевой конус плоской фоновой метрики границей эффективной искривленной метрики $g_{\mu \nu}$. Плоская метрика является наблюдаемой, так что нарушение нулевого конуса для $\eta_{\mu \nu}$ означает обратную причинность в некоторых лоренцевых системах отсчета, что обычно отвергается. Причинность в теориях высших спинов ранее уже создавала трудности в случае поля спина $3 / 2$. Таким образом Вело и Цванцигер [51] пришли к выводу, что “основной урок, который следует усвоить из нашего анализа, состоит в том, что специальная теория относительности не оказывается автоматически выполненной при записи ковариантно преобразующихся уравнений. Кроме того, решения не должны распространяться быстрее света".

Приводился аргумент о том, что массивная гравитация приводит к нарушению причинности в смысле специальной теории относительности (что актуально из-за наблюдаемой плоской метрики [13], [52]). Как справедливо замечает Чугреев [53], необходимо принять во внимание статическое поле источников, если таковые имеются; в космологических моделях для сохранения причинности может оказаться достаточным повсеместное присутствие материи. Однако уж наверняка истина о том, что Вселенная заполнена материей повсюду, содержит в себе элемент неопределенности и не является непоколебимой ${ }^{11)}$. Гравитационное излучение убывает как $1 / r$, а статическое поле локализованных источников убывает как $e^{-m r}$, так что в конце концов излучение начинает преобладать, угрожая правильному соотношению между

11) Действительно, это утверждение, как правило, рассматривается скорее как соглашение, а не как факт даже в космологии, стремящейся описывать наш реальный мир [6]. 
двумя нулевыми конусами. Поскольку желательно рассматривать как физически осмысленные (хотя, по-видимому, и не соответствующие реальному миру) многие решения, в которых материя не повсеместна и которые действительно имеют гравитационное излучение, требуется некая дополнительная стратегия для обеспечения правильного соотношения между нулевыми конусами двух метрик. Единственный вариант, приходящий на ум, - это внедрить искусственный калибровочный произвол, возможно, с использованием параметризации в духе работ [52], [54], а далее использовать ту же стратегию, что и при наложении $\eta$-причинности в безмассовом случае [13]. Получающаяся калибровочная массивная гравитация имеет группоид, а не группу калибровочных преобразований. Стоит отметить, что параметризация дает результат, который в членах низшего порядка напоминает трюк Штюкельберга при внесении калибровочной свободы в массивный электромагнетизм Прока. Трюк Штюкельберга временами использовался в низшем порядке по гравитационному полю [55], но оставалось неясным, каким же может быть обобщение на нелинейные полевые уравнения. Кажется правдоподобным, что другие методы внедрения искусственной калибровочной свободы, такие как процедура Баталина-ФрадкинаТютина [56] или расфиксирование калибровки [57], должны давать похожие результаты, хотя тщательно эти вопросы мы не исследовали.

Благодарности. Дж. Б. Питтс благодарит К. Брэдинг за помощь в изысканиях, связанных с историей использования Эйнштейном закона сохранения энергии и связанных с этим принципов в его поиске уравнений гравитационного поля. Авторы выражают благодарность за обсуждения Ю. В. Чугрееву, С. Дезеру, А. А. Логунову, Т. Падманабану и М. Виссери, не все из которых согласны со всем тем, что здесь было сказано.

\section{Список литературы}

[1] The Collected Papers of Albert Einstein. Vol. 8. The Berlin Years: Correspondence, 19141918, eds. R. Schulmann, A. J. Kox, M. Janssen, J. Illy, Princeton Univ. Press, Princeton, NJ, 1998; The Collected Papers of Albert Einstein. Vol. 4. The Swiss Years: Writings, 1912-1914, eds. A. Beck, D. Howard, Princeton Univ. Press, Princeton, NJ, 1996; M. Janssen, Ann. Phys. (8), 14:S1 (2005), 58.

[2] M. Janssen, J. Renn, "Untying the knot: How Einstein found his way back to field equations discarded in the Zurich notebook", The Genesis of General Relativity: Sources and Interpretations. Vol. 2. Einstein's Zurich Notebook: Commentary and Essays, Boston Stud. Phil. Sci., 250, ed. J. Renn, Springer (to appear); http://www.tc.umn.edu/ janss011/pdf\%20files/knot.pdf.

[3] M. Fierz, W. Pauli, Proc. Roy. Soc. London, Ser. A, 173 (1939), 211; N. Rosen, Phys. Rev., 57 (1940), 147; 150; A. Papapetrou, Proc. Roy. Irish Acad. Sect. A, 52 (1948), 11; S. N. Gupta, Phys. Rev., 96 (1954), 1683.

[4] R. H. Kraichnan, Phys. Rev., 98 (1955), 1118.

[5] M. Kohler, Z. Phys., 134 (1953), 286.

[6] R. P. Feynman, F. B. Morinigo, W. G. Wagner, B. Hatfield, J. Preskill, K. S. Thorne, Feynman Lectures on Gravitation, Addison-Wesley, Reading, MA, 1995 (original by California Institute of Technology, 1963).

[7] V.I. Ogievetsky, I. V. Polubarinov, Ann. Phys., 35 (1965), 167. 
[8] S. Weinberg, Phys. Rev., 138 (1965), B988.

[9] S. Deser, Gen. Relativity Gravitation, 1 (1970), 9; gr-qc/0411023.

[10] L. P. Grishchuk, A. N. Petrov, A. D. Popova, Commun. Math. Phys., 94 (1984), 379.

[11] А. А. Логунов, М. А. Мествиришвили, ТМФ, 86:1 (1991), 3.

[12] J. B. Pitts, W. C. Schieve, Gen. Relativity Gravitation, 33 (2001), 1319; gr-qc/0101058.

[13] J. B. Pitts, W. C. Schieve, Found. Phys., 34 (2004), 211; gr-qc/0406102.

[14] N. Boulanger, M. Esole, Classical Quantum Gravity, 19 (2002), 2107; gr-qc/0110072.

[15] S. Deser, Classical Quantum Gravity, 4 (1987), L99.

[16] N. Boulanger, T. Damour, L. Gualtieri, M. Henneaux, Nucl. Phys. B, 597 (2001), 127; hep-th/0007220.

[17] S. V. Babak, L. P. Grishchuk, Phys. Rev. D, 61 (2000), 024038 ; gr-qc/9907027.

[18] N. Pinto-Neto, P. I. Trajtenberg, Braz. J. Phys., 30:1 (2000), 181.

[19] T. Padmanabhan, From gravitons to gravity: myths and realit, gr-qc/0409089.

[20] M. Visser, Gen. Relativity Gravitation, 30 (1998), 1717; gr-qc/9705051.

[21] S. V. Babak, L. P. Grishchuk, Int. J. Mod. Phys. D, 12 (2003), 1905; gr-qc/0209006.

[22] И. В. Тютин, Е. С. Фрадкин, ЯФ, 15 (1972), 597.

[23] P. G. O. Freund, A. Maheshwari, E. Schonberg, Astrophys. J., 157 (1969), 857.

[24] D. G. Boulware, S. Deser, Phys. Rev. D, 6 (1972), 3368.

[25] W. Israel, Differential Forms in General Relativity, 2nd ed., Dublin Institute for Advanced Studies, Dublin, 1979.

[26] P. van Nieuwenhuizen, Nucl. Phys. B, 60 (1973), 478.

[27] P. Havas, "Energy-momentum tensors in special and general relativity", Developments in General Relativity, Astrophysics, and Quantum Theory, A Jubilee Volume in Honour of Nathan Rosen, eds. F. I. Cooperstock, L. P. Horwitz, J. Rosen, IOP, Bristol; Israel Phys. Soc., Jerusalem, 1990, 131.

[28] E. Kretschmann, Ann. Phys. (8), 53 (1917), 575.

[29] J. L. Anderson, Principles of Relativity Physics, Academic, New York, 1967.

[30] M. Friedman, Foundations of Space-Time Theories: Relativistic Physics and Philosophy of Science, Princeton Univ. Press, Princeton, N. J., 1983.

[31] J. D. Norton, Erkenntnis, 42 (1995), 223.

[32] Ч. Мизнер, К. Торн, Дж. Уилер, Гравитация, Мир, М., 1977.

[33] J. B. Pitts, Stud. Hist. Philos. Sci. B. Stud. Hist. Philos. Modern Phys., 37 (2006), 347; gr-qc/0506102.

[34] D. Gullini, "Some remarks on the notions of general covariancve and background independence", Approaches to Fundamental Physics: An Assessment of Current Theoretical Ideas, Lect. Notes Phys., 721, eds. E. Seiler, I.-O. Stamatescue, Springer, New York, 2007; gr-qc/0603087.

[35] P. Bergmann, "Topics in the theory of general relativity", Lectures in Theoretical Physics, Brandeis University Summer Institute in Theoretical Physics, Benjamin, New York, 1957, notes by N. A. Wheeler; R. U. Sexl, Fortschr. Phys., 15 (1967), 269; J. Stachel, "The meaning of general covariance: The hole story", Philosophical Problems of the Internal and External Worlds, Essays on the Philosophy of Adolf Grünbaum, eds. J. Earman, A. I. Janis, G. J. Massey, N. Rescher, Univ. of Pittsburgh, Pittsburgh; Universitätsverlag, Konstanz, 1993, 129.

[36] M. J. Gotay, J.E. Marsden, "Stress-energy-momentum tensors and the BelinfanteRosenfeld formula", Mathematical Aspects of Classical Field Theory (Seattle, 1991), Contemp. Math., 132, eds. M. J. Gotay, J. E. Marsden, V. Moncrief, Amer. Math. Soc., Providence, RI, 1992, 367; http://www.math.hawaii.edu/〜 gotay/SEMTensors.pdf.

[37] В. И. Огиевецкий, И. В. Полубаринов, ЖЭТФ, 48 (1965), 1625; Р. Ф. Билялов, Изв. вузов. Сер. матем., 46:11 (2002), 8. 
[38] L. Rosenfeld, Acad. Roy. Belgique. Cl. Sci. Mém., 18:6 (1940), 1; English transl.: Selected Papers of Léon Rosenfeld, eds. R. S. Cohen, J. J. Stachel, Reidel, Dordrecht, 1979.

[39] N. Rosen, "Flat space and variational principle", Perspectives in Geometry and Relativity, Essays in Honor of Vácclav Hlavatý, ed. B. Hoffmann, Indiana Univ., Bloomington, 1966, 325; Gen. Relativity Gravitation, 4 (1973), 435; R. D. Sorkin, Mod. Phys. Lett. A, 17 (2002), 695; http://philsci-archive.pitt.edu/archive/00000565/.

[40] R. M. Wald, General Relativity, Univ. of Chicago, Chicago, 1984.

[41] E. R. Huggins, Quantum Mechanics of the Interaction of Gravity with Electrons: Theory of a Spin-Two Field Coupled to Energy, PhD thesis, California Institute of Technology, Pasadena, 1962.

[42] A. N. Petrov, J. Katz, Roy. Soc. London Proc. Ser. A, 458 (2002), 319; gr-qc/9911025.

[43] M. G. Hare, Canad. J. Phys., 51 (1973), 431; A. S. Goldhaber, M. M. Nieto, Phys. Rev. D, 9 (1974), 1119; L. S. Finn, P. J. Sutton, Phys. Rev. D, 65 (2002), 044022; gr-qc/0109049.

[44] S. Deser, "Bimetric gravity revisited", Developments in General Relativity, Astrophysics, and Quantum Theory, A Jubilee volume in Honour of Nathan Rosen (Jerusalem and Haifa, 1989), Ann. Israel Phys. Soc., 9, eds. F. I. Cooperstock, L. P. Horwitz, J. Rosen, IOP, Bristol, 1990, 77.

[45] В. И. Захаров, Писъма в ЖЭТФ, 12 (1970), 447; H. van Dam, M. Veltman, Nucl. Phys. B, 22 (1970), 397; Gen. Relativity Gravitation, 3 (1972), 215; M. Carrera, D. Giulini, Classical analysis of the van Dam-Veltman discontinuity, gr-qc/0107058.

[46] Ю. М. Лоскутов, ТМФ, 107:2 (1996), 329.

[47] K. Sundermeyer, Constrained Dynamics. With Applications to Yang-Mills Theory, General Relativity, Classical Spin, Dual String Model, Lecture Notes in Phys., 169, Springer, BerlinNew York, 1982.

[48] J. B. Pitts, J. Phys.: Conf. Ser., 33 (2006), 279; hep-th/0601185.

[49] A. Anderson, J. W. York, Jr., Phys. Rev. Lett., 81 (1998), 1154; gr-qc/9807041.

[50] S. S. Gershtein, A. A. Logunov, M. A. Mestvirishvili, On one fundamental property of gravitational field in the field theory, gr-qc/0412122.

[51] G. Velo, D. Zwanziger, Phys. Rev., 186 (1969), 1337.

[52] I. Schmelzer, General ether theorie and graviton mass, gr-qc/9811073.

[53] Ю. В. Чугреев, ТМФ, 138:2 (2004), 349.

[54] N. Arkani-Hamed, H. Georgi, M. D. Schwartz, Ann. Phys., 305 (2003), 96; hep-th/0210184.

[55] R. Delbourgo, A. Salam, Lett. Nuovo Cimento, 12 (1975), 297; S. Hamamoto, Progr. Theor. Phys., 95 (1996), 441; 97 (1997), 141; M. J. Duff, J. T. Liu, H. Sati, Phys. Lett. B, 516 (2001), 156; hep-th/0105008; F. A. Dilkes, M. J. Duff, J. T. Liu, H. Sati, Phys. Rev. Lett., 87 (2001), 041301; hep-th/0102093.

[56] I. A. Batalin, I. V. Tyutin, Int. J. Mod. Phys. A, 6 (1991), 3255.

[57] A. S. Vytheeswaran, Int. J. Mod. Phys. A, 13 (1998), 765; hep-th/9701050.

Поступила в редакцию 11.04.2006, после доработки 25.06.2006 